\title{
Iodine-131 therapy alters the immune/inflammatory responses in the thyroids of patients with Graves' disease
}

\author{
WENHUA DU ${ }^{1}$, QINGYU DONG ${ }^{1}$, XIAOTING LU ${ }^{2}$, XIAOMENG LIU ${ }^{1}$, YUELI WANG ${ }^{1}$, \\ WENXIA LI ${ }^{1}$, ZHENYU PAN $^{3}$, QIAN GONG ${ }^{3}$, CUIGE LIANG ${ }^{1}$ and GUANQI GAO ${ }^{1}$ \\ ${ }^{1}$ Department of Endocrinology, Linyi People's Hospital, Linyi, Shandong 276000; ${ }^{2}$ Department of Clinical Medicine, \\ Taishan Medical University, Taian, Shandong 271000; ${ }^{3}$ Department of Internal Medicine, \\ Shandong Medical College, Linyi, Shandong 276000, P.R. China
}

Received July 11, 2015; Accepted September 1, 2016

DOI: 10.3892/etm.2017.4047

\begin{abstract}
The aim of the present study was to evaluate the serum levels of interleukin-6 (IL-6), CXC chemokine ligand-10 (CXCL-10) and intercellular adhesion molecule-1 (ICAM-1) in patients with Graves' disease (GD) following iodine-131 $\left.{ }^{(131} \mathrm{I}\right)$ therapy. A total of 30 patients with GD participated in the present study. Serum cytokine levels were measured with ELISA, and correlation analyses were performed. Serum levels of IL-6, CXCL-10 and ICAM-1 were significantly higher in patients with GD prior to treatment than those in the control subjects $(\mathrm{P}<0.01)$. Following ${ }^{131} \mathrm{I}$ therapy, the serum levels of IL-6 and CXCL-10 in patients with GD were markedly increased within the first week, gradually decreased to the pretreatment level in the subsequent six months and decreased further at 18 months post-treatment. However, the serum levels of IL-6 and CXCL-10 in patients with GD at 18 months following ${ }^{131}$ I therapy remained significantly higher than in control subjects $(\mathrm{P}<0.01)$. Conversely, serum ICAM-1 levels in patients with GD were gradually increased in the 12 months following ${ }^{131}$ I therapy and reached a relatively stable level thereafter. Furthermore, the Pearson's correlation analysis indicated that the serum levels of IL-6, CXCL-10 and ICAM-1 were not associated with free triiodothyronine, the free thyroxine index, and thyroid-stimulating hormone in these patients. ${ }^{131}$ I therapy was able to alter the immune/inflammatory responses in the thyroids of patients with GD. However, these cytokines (IL-6, CXCL-10, and ICAM-1) are not associated with thyroid function; therefore, they cannot be used as prognostic markers for the ${ }^{131} \mathrm{I}$ therapy of GD.
\end{abstract}

Correspondence to: Dr Guanqi Gao, Department of Endocrinology, Linyi People's Hospital, 27 East Jiefang Road, Linyi, Shandong 276000, P.R. China

E-mail: gaoguanqi2013@126.com

Key words: Graves' disease, iodine-131 therapy, leukin-6, CXC chemokine ligand-10, intercellular adhesion molecule-1

\section{Introduction}

Graves' disease (GD) is an organ-specific autoimmune thyroid disease. The development of GD is influenced by various genetic and environmental factors and the interactions between them (1). GD pathogenesis is generally characterized by abnormalities in T-cell subsets $(2,3)$, innate immune cells (4), and various cytokines, which ultimately decreases immune tolerance and excessively activates B cells, promoting the production of thyroid stimulating hormone (TSH) receptor antibody (TRAb). The biased activation of helper $\mathrm{T}$ (Th) cells has an important role in GD development $(5,6)$ and the imbalance of Th1/Th2 cell immunity may result in a shift toward the Th2-type immune response (7).

At present, iodine-131 $\left({ }^{131} \mathrm{I}\right)$ therapy is the recommended treatment option for GD; however, prognosis estimation remains a critical issue (8). It has been previously reported that the presence of thyroid autoantibodies in patients with GD prior to ${ }^{131}$ I therapy is an independent predictor of the occurrence of hypothyroidism (9). Furthermore, high titers of TRAb in patients with GD prior to ${ }^{131} \mathrm{I}$ therapy are associated with treatment failure, whereas elevated TRAb following ${ }^{131}$ I therapy predicts the occurrence of hypothyroidism (10). However, previous studies have indicated that the incidence of hypothyroidism following ${ }^{131}$ I therapy is not affected by the changes in thyroid autoantibody levels prior to treatment $(11,12)$. Therefore, the association between the level of thyroid autoantibodies and the prognosis of GD following ${ }^{131} \mathrm{I}$ therapy remains controversial. Previous studies on GD have predominantly focused on the abnormalities of immune cells and cytokines. Particularly, as markers of immune responses, cytokines have been intensively investigated $(13,14)$. However, few studies have been conducted to assess the cytokine levels in patients with GD following ${ }^{131}$ I therapy $(15,16)$.

In the present study, 30 patients with GD who were currently receiving ${ }^{131}$ I therapy were followed-up for 18 months. Serum cytokine levels were detected in these patients, including interleukin (IL)-6, CXC chemokine ligand (CXCL)-10 and intercellular adhesion molecule (ICAM)-1. Furthermore, the associations of these cytokine levels with the serum levels of free triiodothyronine (FT3), free thyroxine index (FT4) and thyroid-stimulating hormone (TSH) were also evaluated. 


\section{Materials and methods}

Study subjects. A total of 30 patients with GD (the patient group), who were admitted to the Linyi People's Hospital (Shandong, China) between August 2010 and August 2012, participated in the present study. These patients had been diagnosed with GD according to the diagnostic criteria from the Guidelines for Diagnosis and Treatment of Thyroid Diseases in China (2007) (17). Patients with other autoimmune diseases, hepatic or renal insufficiency, acute or chronic infection, or thyroid-related eye diseases were excluded. No patients had been, or were currently being treated with glucocorticoids or other immunosuppressants. The ${ }^{131} \mathrm{I}$ treatment dosage was calculated individually for each patient, according to the Marinelli formula: Treatment dose $=$ [thyroid mass $(\mathrm{g})$ x 0.1 $(\mathrm{mCi} / \mathrm{g})]$ / [radioactive iodine uptake U24 $\left.\left({ }^{131} \mathrm{I}\right)\right]$. A further 30 gender- and age-matched subjects were used as the control group. Prior written and informed consent were obtained from every patient and the methodology was approved by the Ethics Review Board of the Linyi People's Hospital.

Laboratory measurements. Fasting venous blood (10 $\mu \mathrm{l})$ was collected into sodium citrate tubes from each control subject and patient with GD prior to ${ }^{131} \mathrm{I}$ therapy, after 1 week, and $1,2,3,6,12$ and 18 months following treatment. Blood samples were centrifuged at 2,500 x $g$ for $15 \mathrm{~min}$. Serum was separated and stored at $-80^{\circ} \mathrm{C}$ prior to laboratory tests. Serum concentrations of FT3, FT4, TSH and thyroid peroxidase antibodies (TPOAb) were measured via chemiluminescence assay (Unicel Dxi800) and serum TRAb concentration was measured via radioreceptor assay using commercial kits (all Beckman Coulter, Inc., Brea, CA, USA), according to the manufacturer's protocols. Serum levels of IL-6, CXCL-10 and ICAM-1 were measured using ELISA kits (R\&D Systems, Inc., Minneapolis, MN, USA), according to the manufacturer's protocol.

Statistical analysis. Data were expressed as mean \pm standard deviation. Statistical analysis was performed using the SPSS 19.0 software package (IBM SPSS, Armonk, NY, USA). The $\chi^{2}$ test was used for count data, Student's $t$-test for differences between dependent variables, and one-way analysis of variance for in-group comparisons, with Student-Newman-Keuls and Fisher's least significant difference tests. Pearson's correlation was used for association analysis. $\mathrm{P}<0.05$ was considered to indicate a statistically significant difference.

\section{Results}

Demographic and clinical features of patients with GD prior to ${ }^{131}$ I therapy. Demographic and clinical features of $30 \mathrm{control}$ subjects and 30 patients with GD prior to ${ }^{131} \mathrm{I}$ therapy are listed in Table I. No significant differences in gender and age were found between the patient group and control subjects $(\mathrm{P}>0.05)$. Serum levels of FT3, FT4, TSH, TPOAb and TRAb in the patient group prior to ${ }^{131} \mathrm{I}$ treatment were significantly higher than those in the control group $(\mathrm{P}<0.01)$. Serum levels of IL-6, CXCL-10 and ICAM-1 in the patient group prior to ${ }^{131} \mathrm{I}$ therapy were also significantly higher than those in the control group $(\mathrm{P}<0.01)$. These results demonstrated the significant immune/inflammatory responses in patients with GD prior to treatment.

Changes in serum levels of IL-6, CXCL-10, and ICAM-1 in patients with $G D$ following ${ }^{131} I$ therapy. Serum levels of IL-6, CXCL-10, and ICAM-1 were determined in the patient group at the indicated time points following ${ }^{131} \mathrm{I}$ therapy and compared with the before treatment level and corresponding control group. As presented in Fig. 1, in the patient group, the serum IL-6 level increased rapidly following ${ }^{131} \mathrm{I}$ therapy, peaking at 1 week post-treatment $(\mathrm{P}<0.01)$ and subsequently declining. Serum levels of IL-6 in the patient group at 12 and 18 months following ${ }^{131} \mathrm{I}$ therapy were significantly lower than the before-treatment value $(\mathrm{P}<0.05)$, although they remained significantly higher than those in the control group $(\mathrm{P}<0.01)$. Furthermore, as presented in Fig. 2, the serum level of CXCL-10 was also significantly elevated in the patient group at 1 week following ${ }^{131} \mathrm{I}$ therapy $(\mathrm{P}<0.01)$. In the subsequent six months, serum CXCL-10 levels in the patient group gradually declined, to a level comparable with the before-treatment value. At 12 and 18 months post-treatment, serum CXCL-10 levels had declined further and were significantly lower than the before-treatment value $(\mathrm{P}<0.05)$; however, they remained significantly higher than those in the control group $(\mathrm{P}<0.01)$. Conversely, the serum ICAM-1 level exhibited a less-marked increase in the patient group in the first week following ${ }^{131} \mathrm{I}$ therapy, which continuously increased over the next 12 months $(\mathrm{P}<0.05)$ and a relatively stable level was achieved thereafter (Fig. 3). These results suggest that the inflammatory response is maintained in the patients with GD following ${ }^{131} \mathrm{I}$ therapy.

The present results from the Pearson's correlation analysis demonstrated that serum levels of IL-6, CXCL-10, and ICAM-1 were not associated with FT3, FT4 and TSH in the patient group (Table II). These results suggest that changes in the serum levels of IL-6, CXCL-10 and ICAM-1 in patients with GD following ${ }^{131} \mathrm{I}$ therapy may be associated with changes in immune/inflammatory responses, rather than alterations in thyroid function.

\section{Discussion}

GD is a common organ-specific autoimmune disease (1). Abnormalities in T-cell subsets (2) and other innate immune cells (4), and the production of TRAb due to excessively activated B cells (18), are important pathogenic events in GD. As one of the most commonly used treatments for GD, ${ }^{131} \mathrm{I}$ therapy is able to induce cellular apoptosis, which may induce the rupture of thyroid follicular cells and induce the release of cellular contents into the bloodstream. During this process, antibody production may be enhanced by antigens released by the rupture of thyroid follicular cells. Furthermore, the immune response and inflammatory reactions in the thyroid may be altered by the ionizing radiation.

Various cytokines have been determined to be associated with the pathology of GD, including ILs, tumor necrosis factor- $\alpha$, interferon- $\gamma$ and RANTES, which are markers for immune and inflammatory responses $(13,19,20)$. Despite intensive investigation, few cytokines have been confirmed to be altered in patients with GD following ${ }^{131} \mathrm{I}$ therapy $(15,16)$. It has been demonstrated previously that IL-6 (21), CXCL-10 (22) 
Table I. Demographic and clinical characteristics of patients with GD prior to iodine-131 therapy and control subjects.

\begin{tabular}{|c|c|c|c|}
\hline Characteristic & $\begin{array}{c}\text { Controls } \\
n=30\end{array}$ & $\begin{array}{l}\text { Patients with GD } \\
\qquad n=30\end{array}$ & $\mathrm{P}$-value \\
\hline Gender & 4 males/26 females & 5 males $/ 25$ females & 0.74 \\
\hline Age (years) & $35.72 \pm 3.16$ & $36.12 \pm 2.34$ & 0.33 \\
\hline FT3 $(\mathrm{mmol} / \mathrm{l})$ & $4.82 \pm 1.28$ & $20.75 \pm 6.79$ & $<0.01$ \\
\hline FT4 (mmol/l) & $16.72 \pm 3.49$ & $42.01 \pm 8.92$ & $<0.01$ \\
\hline TSH (uIU/ml) & $2.34 \pm 1.62$ & $0.01 \pm 0.01$ & $<0.01$ \\
\hline TPOAb (IU/ml) & $32.17 \pm 16.94$ & $479.83 \pm 36.72$ & $<0.01$ \\
\hline $\mathrm{TRAb}(\mathrm{IU} / \mathrm{l})$ & $0.85 \pm 0.36$ & $11.73 \pm 3.95$ & $<0.01$ \\
\hline IL-6 (pg/ml) & $43.70 \pm 5.05$ & $114.93 \pm 12.35$ & $<0.01$ \\
\hline CXCL-10 (pg/ml) & $78.07 \pm 8.83$ & $183.67 \pm 18.52$ & $<0.01$ \\
\hline ICAM-1 (pg/ml) & $161.45 \pm 7.64$ & $345.69 \pm 16.83$ & $<0.01$ \\
\hline
\end{tabular}

Data are presented as the mean \pm standard deviation. Bold text indicates a significant difference. GD, Graves' disease; FT3, free tri-iodothyronine; FT4, free thyroxine; TSH, thyroid stimulating hormone; TPOAb, thyroid peroxidase antibody; TRAb, TSH receptor autoantibody; IL, interleukin; CXCL, CXC chemokine ligand; ICAM, intercellular adhesion molecule.

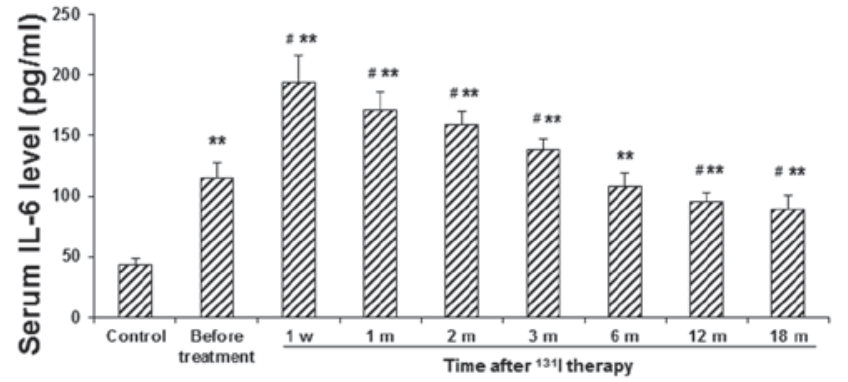

Figure 1. Serum levels of IL-6 in patients with GD before and after ${ }^{131} \mathrm{I}$ therapy. Serum levels of IL-6 in patients with GD were determined using ELISA, prior to ${ }^{131} \mathrm{I}$ therapy, at $1 \mathrm{w}$, and $1,2,3,6,12$ and $18 \mathrm{~m}$ post-treatment. ${ }^{* *} \mathrm{P}<0.01$ vs. the corresponding control group; ${ }^{~} \mathrm{P}<0.05$ vs. the before-treatment level in patients with GD. IL, interleukin; GD, Graves' disease; ${ }^{131} \mathrm{I}$, iodine-131; w, week; m, month.

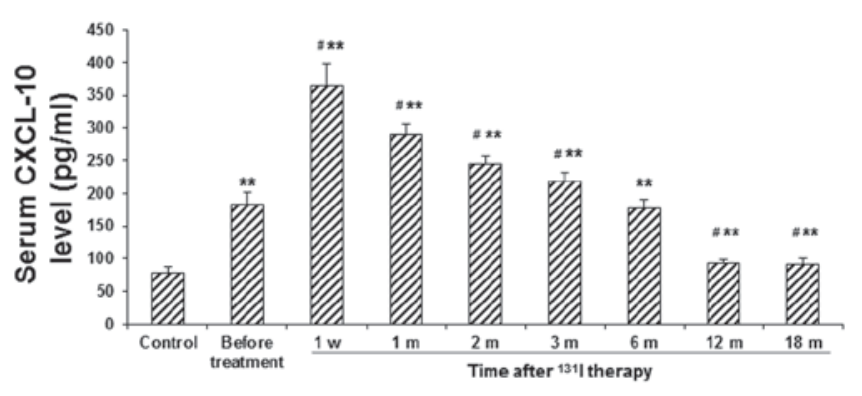

Figure 2. Serum levels of CXCL-10 in patients with GD before and after ${ }^{131}$ I therapy. Serum levels of CXCL-10 in patients with GD were determined using ELISA, prior to ${ }^{131} \mathrm{I}$ therapy, at $1 \mathrm{w}$, and 1,2, 3, 6, 12 and $18 \mathrm{~m}$ post-treatment. ${ }^{* *} \mathrm{P}<0.01$ vs. the corresponding control group; ${ }^{*} \mathrm{P}<0.05$ vs. the before-treatment level in patients with GD. CXCL, CXC chemokine ligand; GD, Graves' disease; ${ }^{131}$ I, iodine-131; w, week; m, month.

and ICAM-1 (23) levels are significantly higher in patients with GD than in healthy subjects, which may be associated with immune/inflammatory responses in the thyroid. However,

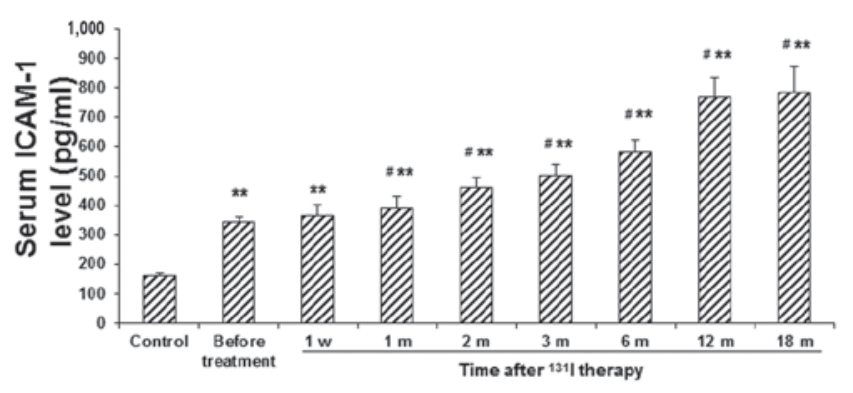

Figure 3. Serum levels of ICAM-1 in patients with GD before and after ${ }^{131} \mathrm{I}$ therapy. Serum levels of ICAM-1 in patients with GD were determined using ELISA, prior to ${ }^{131} \mathrm{I}$ therapy, at $1 \mathrm{w}$, and 1, 2, 3, 6, 12 and 18 m post-treatment. ${ }^{* *} \mathrm{P}<0.01$ vs. the corresponding control group; ${ }^{*} \mathrm{P}<0.05$ vs. the before-treatment level in patients with GD. ICAM, intracellular adhesion molecule; GD, Graves' disease; ${ }^{131}$ I, iodine-131; w, week; m, month.

it has not yet been fully elucidated whether this occurs in patients with GD following ${ }^{131}$ I therapy.

IL-6, as a Th2 cytokine, predominantly regulates B- and T-lymphocyte functions (24). In patients with GD, IL-6 is produced by infiltrating immune cells and thyrocytes (25). Serum IL-6 levels in patients with GD have been reported to be significantly higher than those in healthy controls $(21,26)$. However, the clinical significance of serum IL-6 levels remains controversial. Wahrenberg et al (21) have determined that the serum level of IL- 6 indicates the tendency of inflammatory response. Furthermore, Heuer et al (26) claim that the elevated IL- 6 concentration may reflect disease severity. In the present study, it was demonstrated that serum IL-6 levels in patients with GD were significantly higher than those in the control group. The serum IL-6 level increased and peaked rapidly following ${ }^{131} \mathrm{I}$ therapy, and subsequently returned to the before-treatment level within six months, prior to declining further by 18 months post-treatment. Pearson's correlation analysis demonstrated that changes in serum IL-6 levels in the patient group following ${ }^{131}$ I therapy were not correlated with thyroid function, suggesting that the changes in serum IL-6 
Table II. Correlation analysis of serum levels of IL-6, CXCL-10, and ICAM-1 with FT3, FT4, and TSH in patients with GD and control subjects.

\begin{tabular}{lcllr}
\hline Cytokine & Statistical value & FT3 & FT4 & TSH \\
\hline IL-6 & $\mathrm{R}$ & 0.037 & 0.046 & -0.018 \\
& P-value & 0.61 & 0.54 & 0.61 \\
CXCL-10 & $\mathrm{R}$ & 0.072 & 0.063 & -0.014 \\
& P-value & 0.24 & 0.29 & 0.61 \\
ICAM-1 & $\mathrm{R}$ & 0.047 & 0.053 & -0.061 \\
& P-value & 0.36 & 0.31 & 0.23 \\
\hline
\end{tabular}

IL, interleukin; CXCL, CXC chemokine ligand; ICAM, intercellular adhesion molecule; FT3, free tri-iodothyronine; FT4, free thyroxine index; TSH, thyroid stimulating hormone; GD, Graves' disease; R, Pearson's coefficient.

may be associated with changes in immune/inflammatory responses. Conversely, the present results indicated that the serum level of IL-6 in the patient group at 18 months following ${ }^{131}$ I therapy was significantly higher than in the control group, suggesting a marked inflammatory response in the thyroid following ${ }^{131} \mathrm{I}$ therapy. Further studies are required to clarify whether this is related to the thyroid dysfunction following ${ }^{131} \mathrm{I}$ therapy.

CXCL-10, also known as interferon- $\gamma$-inducible protein 10 (IP-10), is associated with the infiltration, localization and activation of lymphocytes in the thyroid (27). In addition to endothelial and T cells, CXCL-10 is predominately secreted by the thyroid cells in inflammatory responses, which contributes to the pathogenesis of GD $(22,28)$. The present results demonstrated that serum CXCL-10 levels in the patient group were significantly higher than those in the control group, which is in accordance with previous findings $(22,28)$. Furthermore, the serum level of CXCL-10 in the patient group rapidly increased following ${ }^{131}$ I therapy and subsequently declined to the before-treatment level within six months, with a significantly lower level at 18 months post-treatment, while remaining significantly higher that of the control group. These results were consistent with those observed for the serum IL-6 levels. CXCL-10 and IL-6 mediate the Th1/Th2 responses; therefore, these findings suggest that ${ }^{131} \mathrm{I}$ therapy for GD may significantly enhance cellular and humoral immune/inflammatory responses.

ICAM-1, which is a member of the adhesion molecule immunoglobulin superfamily, has an important role in lymphocyte adhesion, T-cell activation and the antigen-presenting process, which indicates non-specific inflammation (29). In accordance with a previous study (23), the present results demonstrated that the serum ICAM-1 level in the patient group was significantly higher than that of the control group. Serum ICAM-1 levels have been observed to be altered by treatment with anti-thyroid drugs (30), suggesting that the inflammatory responses in patients with GD may be alleviated by these drugs. Furthermore, the present results demonstrated that the serum levels of ICAM-1 in patients with GD were gradually increased during the 18 months following ${ }^{131}$ I therapy, suggesting inflammatory activity. This phenomenon is markedly different from the changes observed in the serum levels of IL- 6 and CXCL-10. As ICAM-1 is a marker for the activities of endothelial cells and fibroblasts (31), the continuously elevated ICAM-1 level following ${ }^{131}$ I therapy may be associated with the involvement of endothelial cells and fibroblasts in the repair of the thyroid.

In conclusion, the present results demonstrated that serum levels of IL-6, CXCL-10 and ICAM-1 were significantly elevated in patients with GD prior to treatment, and that ${ }^{131} \mathrm{I}$ therapy was able to alter the serum cytokine levels in these patients. However, the changes in serum levels of IL-6, CXCL-10 and ICAM-1 were not associated with thyroid function. Furthermore, no data regarding the thyroid autoantibodies prior to and following the ${ }^{131}$ I therapy in these patients was obtained in the present study, and the relationship between thyroid autoantibody changes and cytokine alterations cannot be evaluated. Therefore, these findings suggest that these cytokines cannot be used as prognostic markers for the ${ }^{131}$ I therapy of GD.

\section{Acknowledgements}

This work was supported by the National Natural Science Foundation of China (grants nos. 30971595 and 30771017), Natural Science Foundation of Shandong Province, China (grant no. ZH2011HM067), and the Medical and Health Science Technology Development Program of Shandong Institute (grant no. 2011HW023).

\section{References}

1. Prummel MF, Strieder T and Wiersinga WM: The environment and autoimmune thyroid diseases. Eur J Endocrinol 150: 605-618, 2004.

2. Saitoh O, Abiru N, Nakahara M and Nagayama Y: $\mathrm{CD}^{+} \mathrm{CD} 122^{+}$ $\mathrm{T}$ cells, a newly identified regulatory $\mathrm{T}$ subset, negatively regulate Graves' hyperthyroidism in a murine model. Endocrinology 148: 6040-6046, 2007.

3. Hammerstad SS, Jahnsen FL, Tauriainen S, Hyöty H, Paulsen T, Norheim I and Dahl-Jørgensen K: Immunological changes and increased expression of myxovirus resistance protein a in thyroid tissue of patients with recent onset and untreated Graves' disease. Thyroid 24: 537-544, 2014.

4. Kawashima A, Tanigawa K, Akama T, Yoshihara A, Ishii N and Suzuki K: Innate immune activation and thyroid autoimmunity. J Clin Endocrinol Metab 96: 3661-3671, 2011.

5. Kidd P: Th1/Th2 balance: The hypothesis, its limitations and implications for health and disease. Altern Med Rev 8: 223-246, 2003. 
6. Rapoport B and McLachlan SM: Graves' hyperthyroidism is antibody-mediated but is predominantly a Th1-type cytokine disease. J Clin Endocrinol Metab 99: 4060-4061, 2014.

7. Morshed SA, Latif R and Davies TF: Delineating the autoimmune mechanisms in Graves' disease. Immunol Res 54: 191-203, 2012.

8. Bahn Chair RS, Burch HB, Cooper DS, Garber JR, Greenlee MC, Klein I, Laurberg P, McDougall IR, Montori VM, Rivkees SA, et al Hyperthyroidism and other causes of thyrotoxicosis: Management guidelines of the American Thyroid Association and American Association of Clinical Endocrinologists. Thyroid 21: 593-646, 2011

9. Ahmad AM, Ahmad M and Young ET: Objective estimates of the probability of developing hypothyroidism following radioactive iodine treatment of thyrotoxicosis. Eur J Endocrinol 146: 767-775, 2002.

10. Chiovato L, Fiore E, Vitti P, Rocchi R, Rago T, Dokic D, Latrofa F Mammoli C, Lippi F, Ceccarelli C and Pinchera A: Outcome of thyroid function in Graves' patients treated with radioiodine: Role of thyroid-stimulating and thyrotropin-blocking antibodies and of radioiodine-induced thyroid damage. J Clin Endocrinol Metab 83: 40-46, 1998.

11. Andrade VA, Gross JL and Maia AL: The effect of methimazole pretreatment on the efficacy of radioactive iodine therapy in Graves hyperthyroidism: One-year follow-up of a prospective, randomized study. J Clin Endocrinol Metab 86: 3488-3493, 2001.

12. Gómez-Arnaiz N, Andía E, Gumà A, Abós R, Soler J and Gómez JM: Ultrasonographic thyroid volume as a reliable prognostic index of radioiodine-131 treatment outcome in Graves' disease hyperthyroidism. Horm Metab Res 35: 492-497, 2003.

13. Mikos H, Mikos M, Obara-Moszynska M and Niedziela M: The role of the immune system and cytokines involved in the pathogenesis of autoimmune thyroid disease (AITD). Endokrynologia Polska 65: 150-155, 2014.

14. Khan FA, Al-Jameil N, Khan MF, Al-Rashid M and Tabassum H: Thyroid dysfunction: An autoimmune aspect. Int J Clin Exp Med 8: 6677-6681,2015.

15. Dong QY, Li SJ, Gao GQ, Liu XM, Li WX, Liang CG, Du WH and Wsng YL: Short-term effect of radioactive iodine therapy on CXCL-10 production in Graves' disease. Clin Invest Med 34: E262, 2011.

16. Jurgilewicz DH, Rogowski F, Łebkowska U, Citko A, Jaroszewicz E and Parfieńczyk A: E-selectin, L-selectin, ICAM-1 and IL-6 concentrations changes in the serum of patients with hyperthyroidism in the early period of radioiodine I-131 therapy. Nucl Med Rev Cent East Eur 5: 39-42, 2002

17. Chinese Society of Endocrinology: Medical guidelines for the management of Thyroid disease in China. Clin J Intern Med, 46 (No. 10, 2007)

18. Zeki K, Tanaka Y, Fujihira T, Watanabe K, Suzuki H, Yamashita U and Eto S: Immunological abnormality of peripheral blood B cells in patients with autoimmune thyroid disease. Endocrinol Jpn 36 335-342, 1989.

19. Mysliwiec J, Palyga I, Nikolajuk A, Kowalska A and Gorska M: Serum interleukin-16 and RANTES during treatment of Graves orbitopathy with corticosteroids and teleradiotherapy. Endokrynol Pol 63: 92-96, 2012.
20. Niyazoglu M, Baykara O, Koc A, Aydoğdu P, Onaran I, Dellal FD, Tasan E and Sultuybek GK: Association of PARP-1, NF- $\kappa$ B, NF- $\kappa$ BIA and IL-6, IL- $1 \beta$ and TNF- $\alpha$ with Graves Disease and Graves Ophthalmopathy. Gene 547: 226-232, 2014.

21. Wahrenberg $\mathrm{H}$, Wennlund $\mathrm{A}$ and Hoffstedt J: Increased adipose tissue secretion of interleukin-6, but not of leptin, plasminogen activator inhibitor-1 or tumour necrosis factor alpha, in Graves' hyperthyroidism. Eur J Endocrinol 146: 607-611, 2002

22. Antonelli A, Rotondi M, Fallahi P, Romagnani P, Ferrari SM, Buonamano A, Ferrannini E and Serio M: High levels of circulating CXCL chemokine ligand 10 are associated with chronic autoimmune thyroiditis and hypothyroidism. J Clin Endocrinol Metab 89: 5496-5499, 2004.

23. Arao T, Morimoto I, Kakinuma A, Ishida O, Zeki K, Tanaka Y, Ishikawa $\mathrm{N}$, Ito $\mathrm{K}$, Ito $\mathrm{K}$ and Eto $\mathrm{S}$ : Thyrocyte proliferation by cellular adhesion to infiltrating lymphocytes through the intercellular adhesion molecule-1/lymphocyte function-associated antigen-1 pathway in Graves' disease. J Clin Endocrinol Metab 85: 382-389, 2000.

24. Hunter CA and Jones SA: IL-6 as a keystone cytokine in health and disease. Nat Immunol 16: 448-457, 2015.

25. Salvi M, Girasole G, Pedrazzoni M, Passeri M, Giuliani N, Minelli R, Braverman LE and Roti E: Increased serum concentrations of interleukin-6 (IL-6) and soluble IL-6 receptor in patients with Graves' disease. J Clin Endocrinol Metab 81: 2976-2979, 1996.

26. Heuer M, Aust G, Ode-Hakim S and Scherbaum WA: Different cytokine mRNA profiles in Graves' disease, Hashimoto's thyroiditis and nonautoimmune thyroid disorders determined by quantitative reverse transcriptase polymerase chain reaction (RT-PCR). Thyroid 6: 97-106, 1996.

27. Antonelli A, Rotondi M, Ferrari SM, Fallahi P, Romagnani P, Franceschini SS, Serio M and Ferrannini E: Interferon-gamma-inducible alpha-chemokine CXCL10 involvement in Graves' ophthalmopathy: Modulation by peroxisome proliferator-activated receptor-gamma agonists. J Clin Endocrinol Metab 91: 614-620, 2006.

28. Antonelli A, Ferrari SM, Corrado A, Di Domenicantonio A and Fallahi P: Autoimmune thyroid disorders. Autoimmun Rev 14: 174-180, 2015.

29. Makgoba MW, Sanders ME, Ginther Luce GE, Dustin ML, Springer TA, Clark EA, Mannoni P and Shaw S: ICAM-1 a ligand for LFA-1-dependent adhesion of $\mathrm{B}, \mathrm{T}$ and myeloid cells. Nature 331: 86-88, 1988.

30. Wenisch C, Myskiw D, Parschalk B, Hartmann T, Dam K and Graninger W: Soluble endothelium-associated adhesion molecules in patients with Graves' disease. Clin Exp Immunol 98: 240-244, 1994.

31. Crescioli C, Cosmi L, Borgogni E, Santarlasci V, Gelmini S, Sottili M, Sarchielli E, Mazzinghi B, Francalanci M, Pezzatini A et al: Methimazole inhibits CXC chemokine ligand 10 secretion in human thyrocytes. J Endocrinol 195: 145-155, 2007. 